\title{
Synergistic Interactions in Mixed Micelles of Cationic 14-s-14 Gemini with Conventional Surfactants: Spacer and Counterion Effects
}

\author{
AZUM Naved NAQVI Andleeb Z. AKRAM Mohd. KABIR-UD-DIN * \\ (Department of Chemistry, Aligarh Muslim University, Aligarh-202002, India)
}

\begin{abstract}
The mixed micelle formation of binary cationic 14-s-14 gemini with conventional single chain surfactants was studied by conductivity measurements. The critical micelle concentration $(\mathrm{cmc})$ and the degree of counterion binding values $(g)$ of the binary systems were determined. The results were analyzed by applying regular solution theory (RST) to calculate micellar compositions $(X)$, activity coefficients $\left(f_{1}, f_{2}\right)$, and the interaction parameters $(\beta)$. The synergistic interactions of all the investigated cationic gemini+conventional surfactant combinations were found to be dependent upon the length of hydrophobic spacer of the gemini surfactant. The excess Gibbs free energy of mixing was evaluated, and it indicated relatively more stable mixed micelles for the binary combinations.
\end{abstract}

Key Words: Mixed micelle; Gemini surfactant; Conventional surfactant; Critical micelle concentration; Degree of counterion binding; Synergistic interaction

Gemini surfactants are a special class of $\operatorname{surfactants}^{[1-3]}$, which contain each of hydrophobic and hydrophilic groups coupled together via a spacer of varied nature (hydrophobic, hydrophilic, flexible, or rigid) (Fig.1). These novel surfactants show many unexpected surface properties when compared with conventional counterparts (one hydrophobic and hydrophilic group), i.e., lower critical micelle concentrations, much greater efficiency in reducing the surface tension of water, etc. Since these surfactants contain two hydrophobic tails per molecule, surface activity is enhanced and increases with increasing chain length. Gemini surfactants have manifold applications in the detergent and cosmetic industries due to their enhanced surface activity, emulsifying property, and mildness to skin. Noteworthy among geminis, cationic ones are used to a large extent in a number of pharmaceutical, biomedical, and personal care product applications ${ }^{[4]}$ (due to low mammalian toxicity).

As we know, commercially used surfactants are invariably mixtures of two or more types of surfactants. The mixtures have gained importance in the industrial, pharmaceutical, and biological fields due to their better performance and cost effectiveness over single surfactant. The mixed micelles have also been found to enhance the adsorption of various drugs in the human body ${ }^{[5]}$. Mixture of surfactants is used to facilitate the dissolution and

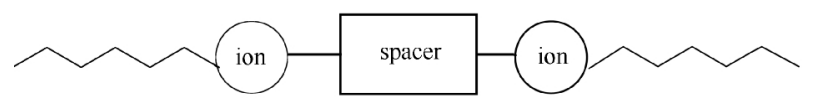

Fig.1 Schematic representation of the structure of the gemini surfactant improved tolerance of water hardness ${ }^{[6]}$. All the above occur due to synergism observed between different components of mixtures. Synergism is defined as the condition in which the properties of a mixture are better than those attainable with the individual components separately. An important mixed system is that including ionic gemini and ionic conventional surfactants with the same charge. Cosmetic industries use mixture of surfactants in low concentrations (synergism in critical micelle concentration) to avoid potential skin irritation ${ }^{[7]}$. The synergism is also beneficial for the environment as it allows the amount of surfactants released and hence their impact to be substantially reduced ${ }^{[8]}$.

Because of better performance and low consumption of mixed systems than pure surfactants, considerable attention has been devoted towards understanding the detailed physicochemical behavior of amphiphiles in the recent years ${ }^{[9-10]}$. Binary mixtures of surfactants have often been studied to investigate the micellar composition, aggregation number, molecular interactions, and free energy of micellization, interfacial adsorption, micro-polarity employing surface tension, electrical conductivity, solubilization, NMR, and fluorescence quenching methods.

Many phenomenological models have been put forward for dealing with the mixed binary systems to evaluate the composition and interaction parameter among the components at the air/water interface and in the micellar phase. A phase separation model, which relates the mole fraction and critical micelle concentration of the $i$ th component in an ideal mixture is known as Clint's model $^{[11-12]}$. Rubingh ${ }^{[13]}$ gave a theoretical model on the basis of regular solution theory (RST) that relates the monomer

Received: November 12, 2009; Revised: March 2, 2010; Published on Web: April 21, 2010.

${ }^{*}$ Corresponding author. Email: kabir7@ rediffmail.com.

The project was supported by the Council of Scientific and Industrial Research (CSIR) Funds, New Delhi, India (ACK. No.: 123075/2K8/1).

C. Editorial office of Acta Physico-Chimica Sinica 
concentration to micellar concentration. This theory was criticized on thermodynamic grounds, although found to be satisfactory in many cases. Rosen et al. ${ }^{[14]}$ extended Rubingh theory to monolayer at the air/water interface. Motomura et al. ${ }^{[15]}$ proposed a mixed micellar model based on thermodynamic considerations. Blankschtein et al. ${ }^{[16-18]}$ have developed a molecular thermodynamic theory to describe the binary and ternary mixed surfactant systems. Maeda ${ }^{[19]}$ has proposed an approach for mixed micelle formation involving nonionic and ionic surfactants based on phase separation model.

This paper aims to investigate molecular interaction in the mixed micellization of cationic gemini alkanediyl- $\alpha, \omega$-bis(tetradecyldimethylammonium bromide), referred to as 14-s-14 $(s=4,5,6)$ and two conventional surfactants (cetylpyridinium bromide and cetylpyridinium chloride). The mixtures are characterized by their critical micelle concentration $(\mathrm{cmc})$ at $303 \mathrm{~K}$. The nature and strength of the interactions between surfactant mixtures were obtained by the calculating the values of their $\beta$ parameters. To assist this experimental analysis, the theoretical model of ideal solutions (Clint's model) and the regular solution approach (Rubingh's model) have been used.

\section{Experimental}

\subsection{Materials}

Gemini surfactants were synthesized by reacting a dibromoalkanes with tetradecyl dimethylamine ${ }^{[20]}$ :

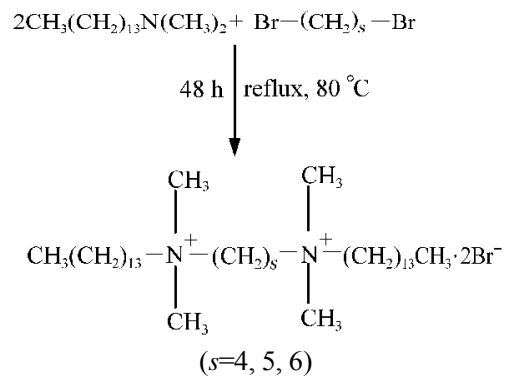

The molar ratio was $2.1: 1$ and the mixture was refluxed in ethanol for $48 \mathrm{~h}$. The solvent was removed and then the raw material was recrystallized in ethanol-ethyl acetate mixtures. The crystallization was normally repeated four times. All products were checked by ${ }^{1} \mathrm{H}$ NMR spectroscopy using $\mathrm{CDCl}_{3}$ as solvent.

Cetylpyridinium bromide (CPB) and cetylpyridinium chloride (CPC) were purchased from Merck and were used without further purification. All solutions were prepared in doubly-distilled water and experiments were performed at $303 \mathrm{~K}$.

\subsection{Method}

The critical micelle concentration (cmc) was determined using conductivity method. The measurements were performed on an ELICO (type CM 82T) bridge equipped with platinized electrodes (cell constant is $1.02 \mathrm{~cm}^{-1}$ ). The experiments were carried out by adding progressively concentrated surfactant stock solution into the thermostated solvent. The critical micellar concentration of the surfactant used was obtained from the plots of specific conductivity $(\kappa)$ as a function of the surfactant concentration. The $\mathrm{cmc}$ value was taken from the intersection of the two straight lines drawn before and after the intersection point in the $\kappa$ vs surfactant concentration plot. Differential conductivity $(\mathrm{d} \kappa / \mathrm{d} c)$ plots in all cases were also used to check the $\mathrm{cmc}$; they are considered to give more accurate $\mathrm{cmc}$ values than the values evaluated only from $\kappa$ plots. The degree of counterion binding $(g)$ was obtained from the ratio of the slopes of the conductivity $v s$ surfactant concentration plots above and below the cmc.

\section{Results}

It is well known that specific conductivity is linearly related to the surfactant concentration $(c)$ in the both premicellar and postmicellar regions and that the intersection point between the two straight lines provides the cmc. Experimental values of specific conductivity $(\kappa)$ are plotted as a function of total surfactant concentration $\left(c_{\mathrm{T}}\right)$ at several constant values of mole fraction $(\alpha)$ (a selected example is shown in Fig.2). The cmc in each case was evaluated by taking the first derivative of the plot. The first derivative is of sigmoidal type and can be adequately described by using a Boltzmann type sigmoid, which has the following analytical expression:

$$
f(x)=\frac{A_{1}-A_{2}}{1+\mathrm{e}^{\left(x-x_{0}\right) / \Delta x}}+A_{2}
$$

where, $A_{1}$ and $A_{2}$ represents the asymptotic value for small and large values of $x, x_{0}$ represents the central point of the transition and $\Delta x$ deals with the width of the transition. The degree of counterion binding of the micelle, $g$, can be obtained from the $A_{2} / A_{1}$ ratio as $g=\left(1-A_{2} / A_{1}\right)$. These values are reported in Tables 1 to 3 . Tables 1-3 list the cmc values obtained at various mole ratios at $303 \mathrm{~K}$, as well as the composition of each mixed system. The variation of $\mathrm{cmc}$ along with ideal $\mathrm{cmc}\left(\mathrm{cmc}^{*}\right)$ vs mole fraction of component 1 (conventional surfactant) are recorded in Tables 1 to 3 and Figs.3, 4 present the same graphically. The parameters $\left(X_{1}, X_{1}^{\text {ideal }}, \beta, f_{1}, f_{2}, \Delta G_{\mathrm{ex}}\right)$, calculated using Rubingh and Motomura models to analyze the mixed micelle formation, are also compiled in Tables 1 to 3 .

\section{Discussion}

Aqueous solutions of the conventional and gemini binary sys-

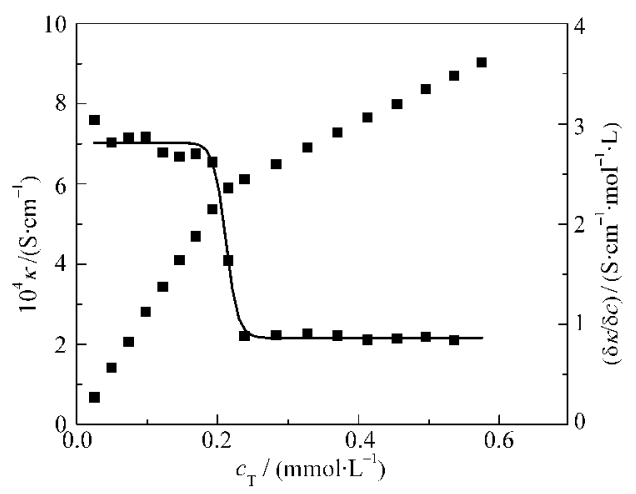

Fig.2 Plots of specific conductivity $(\kappa)$ and first derivative of specific conductivity $(\delta \kappa / \delta c)$ of 14-4-14+CPB versus total surfactant concentration $\left(c_{\mathrm{T}}\right)$ at $\alpha_{\mathrm{CPB}}=0.4$ 
Table 1 Various physicochemical parameters for 14-4-14+CPB/CPC mixed systems at $303 \mathrm{~K}$ (evaluated on the basis of conductivity measurements)

\begin{tabular}{|c|c|c|c|c|c|c|c|c|c|c|}
\hline & $\alpha_{1}$ & $\mathrm{cmc} /\left(\mathrm{mmol} \cdot \mathrm{L}^{-1}\right)$ & $\mathrm{cmc}^{*} /\left(\mathrm{mmol} \cdot \mathrm{L}^{-1}\right)$ & $g$ & $X_{1}$ & $X_{1}^{\text {idcal }}$ & $\beta$ & $f_{1}$ & $f_{2}$ & $\Delta G_{\mathrm{ex}} /\left(\mathrm{J} \cdot \mathrm{mol}^{-1}\right)$ \\
\hline \multirow[t]{6}{*}{$\mathrm{CPB}$} & 0.0 & 0.150 & & 0.68 & & & & & & \\
\hline & 0.2 & 0.171 & 0.177 & 0.70 & 0.085 & 0.0547 & -0.482 & 0.668 & 0.996 & -96 \\
\hline & 0.4 & 0.211 & 0.217 & 0.69 & 0.152 & 0.134 & -0.215 & 0.857 & 0.995 & -70 \\
\hline & 0.6 & 0.241 & 0.278 & 0.65 & 0.312 & 0.258 & -0.708 & 0.715 & 0.933 & -384 \\
\hline & 0.8 & 0.325 & 0.389 & 0.62 & 0.486 & 0.481 & -0.725 & 0.825 & 0.842 & -458 \\
\hline & 1.0 & 0.648 & & 0.67 & & & & & & \\
\hline \multirow[t]{6}{*}{$\mathrm{CPC}$} & 0.0 & 0.150 & & 0.68 & & & & & & \\
\hline & 0.2 & 0.181 & 0.175 & 0.64 & 0.095 & 0.065 & -0.426 & 0.706 & 0.996 & -92 \\
\hline & 0.4 & 0.201 & 0.211 & 0.62 & 0.187 & 0.156 & -0.345 & 0.796 & 0.988 & -132 \\
\hline & 0.6 & 0.254 & 0.265 & 0.61 & 0.310 & 0.294 & -0.197 & 0.910 & 0.981 & -107 \\
\hline & 0.8 & 0.348 & 0.355 & 0.32 & 0.526 & 0.526 & -0.089 & 0.980 & 0.976 & -56 \\
\hline & 1.0 & 0.540 & & 0.69 & & & & & & \\
\hline
\end{tabular}

Table 2 Various physicochemical parameters for 14-5-14+CPB/CPC mixed systems at $303 \mathrm{~K}$ (evaluated on the basis of conductivity measurements)

\begin{tabular}{|c|c|c|c|c|c|c|c|c|c|c|}
\hline & $\alpha_{1}$ & $\mathrm{cmc} /\left(\mathrm{mmol} \cdot \mathrm{L}^{-1}\right)$ & $\mathrm{cmc}^{*} /\left(\mathrm{mmol} \cdot \mathrm{L}^{-1}\right)$ & $g$ & $X_{1}$ & $X_{1}^{\text {ideal }}$ & $\beta$ & $f_{1}$ & $f_{2}$ & $\Delta G_{\mathrm{ex}} /\left(\mathrm{J} \cdot \mathrm{mol}^{-1}\right)$ \\
\hline \multirow[t]{6}{*}{ CPB } & 0.0 & 0.160 & & 0.68 & & & & & & \\
\hline & 0.2 & 0.164 & 0.188 & 0.74 & 0.152 & 0.058 & -1.529 & 0.333 & 0.965 & -497 \\
\hline & 0.4 & 0.178 & 0.229 & 0.67 & 0.260 & 0.141 & -1.573 & 0.423 & 0.899 & -762 \\
\hline & 0.6 & 0.233 & 0.292 & 0.59 & 0.344 & 0.270 & -1.084 & 0.627 & 0.879 & -618 \\
\hline & 0.8 & 0.318 & 0.402 & 0.80 & 0.500 & 0.497 & -0.967 & 0.785 & 0.785 & -609 \\
\hline & 1.0 & 0.648 & & 0.67 & & & & & & \\
\hline \multirow[t]{6}{*}{ CPC } & 0.0 & 0.160 & & 0.68 & & & & & & \\
\hline & 0.2 & 0.170 & 0.186 & 0.69 & 0.135 & 0.069 & -1.019 & 0.466 & 0.982 & -299 \\
\hline & 0.4 & 0.204 & 0.233 & 0.66 & 0.215 & 0.165 & -0.572 & 0.703 & 0.974 & -243 \\
\hline & 0.6 & 0.251 & 0.277 & 0.57 & 0.340 & 0.308 & -0.454 & 0.820 & 0.949 & -257 \\
\hline & 0.8 & 0.333 & 0.366 & 0.59 & 0.536 & 0.542 & -0.385 & 0.920 & 0.895 & -242 \\
\hline & 1.0 & 0.540 & & 0.69 & & & & & & \\
\hline
\end{tabular}

Table 3 Various physicochemical parameters for 14-6-14+CPB/CPC mixed systems at $303 \mathrm{~K}$ (evaluated on the basis of conductivity measurements)

\begin{tabular}{|c|c|c|c|c|c|c|c|c|c|c|}
\hline & $\alpha_{1}$ & $\mathrm{cmc} /\left(\mathrm{mmol} \cdot \mathrm{L}^{-1}\right)$ & $\mathrm{cmc}^{*} /\left(\mathrm{mmol} \cdot \mathrm{L}^{-1}\right)$ & $g$ & $X_{1}$ & $X_{1}^{\text {dical }}$ & $\beta$ & $f_{1}$ & $f_{2}$ & $\Delta G_{\mathrm{ex}} /\left(\mathrm{J} \cdot \mathrm{mol}^{-1}\right)$ \\
\hline \multirow[t]{6}{*}{ CPB } & 0.0 & 0.170 & & 0.63 & & & & & & \\
\hline & 0.2 & 0.165 & 0.199 & 0.70 & 0.178 & 0.062 & -1.852 & 0.286 & 0.943 & -683 \\
\hline & 0.4 & 0.187 & 0.241 & 0.61 & 0.266 & 0.149 & -1.549 & 0.434 & 0.896 & -762 \\
\hline & 0.6 & 0.212 & 0.305 & 0.52 & 0.376 & 0.282 & -1.669 & 0.522 & 0.789 & -988 \\
\hline & 0.8 & 0.300 & 0.415 & 0.38 & 0.510 & 0.512 & -1.332 & 0.726 & 0.707 & -839 \\
\hline & 1.0 & 0.648 & & 0.67 & & & & & & \\
\hline \multirow[t]{6}{*}{ CPC } & 0.0 & 0.170 & & 0.63 & & & & & & \\
\hline & 0.2 & 0.160 & 0.197 & 0.68 & 0.194 & 0.073 & -1.825 & 0.305 & 0.934 & -719 \\
\hline & 0.4 & 0.190 & 0.234 & 0.61 & 0.270 & 0.173 & -1.222 & 0.521 & 0.915 & -607 \\
\hline & 0.6 & 0.232 & 0.289 & 0.54 & 0.376 & 0.321 & -0.969 & 0.685 & 0.872 & -574 \\
\hline & 0.8 & 0.327 & 0.376 & 0.50 & 0.546 & 0.557 & -0.580 & 0.887 & 0.841 & -363 \\
\hline & 1.0 & 0.540 & & 0.69 & & & & & & \\
\hline
\end{tabular}

tems have been characterized through conductivity. We have chosen conductivity because it seemed one of the most straightforward techniques used due to high sensitivity and reproducibility. The surfactants studied herein are cationic surfactants of different chain length and head group. According to Tanford's model ${ }^{[21]}$, the lengthening of surfactant tail by $-\mathrm{CH}_{2}$ clearly affects micellar properties: i.e., the cmc decreases approximately $50 \%$, and the dissociation degree decreases around 25\%. However, change in head group has little effect on micellar properties. The presence of two alkyl chains in 14-s-14 makes the molecule more hydrophobic. The greater the hydrophobicity of the molecule, the greater the distortion of the water structure and the greater the tendency to form micelles, hence the cmc of 14-s14 is about $75 \%$ less than that of CPB and $70 \%$ from that of CPC.

When two or more types of surfactants are mixed together, they form aggregates, but the tendency of aggregation can be different from that of the pure surfactants. Both the ideal and nonideal mixed micelles are possible, the ideality/nonideality in the mixed micelle formation between the cationic gemini and cationic conventional surfactants can be evaluated by using 
Clint's model ${ }^{[11-12]}$, based upon pseudophase thermodynamic formulation. Clint's equation can be used to relate the cmc values as

$$
\frac{1}{\mathrm{cmc}^{*}}=\sum_{i=1}^{n} \frac{\alpha_{i}}{\mathrm{cmc}_{i}}
$$

where $\alpha_{i}$ is the stoichiometric mole fraction in the mixture and $\mathrm{cmc}^{*}$ is the ideal $\mathrm{cmc}$ value of the mixture.

For two surfactants 1 and 2 with cmc values $\mathrm{cmc}_{1}$ and $\mathrm{cmc}_{2}$,

$$
\frac{1}{\mathrm{cmc}^{*}}=\frac{\alpha_{1}}{\mathrm{cmc}_{1}}+\frac{\alpha_{2}}{\mathrm{cmc}_{2}}
$$

This is an idealization which neglects the interaction among different surfactants in the aggregated state and considers the $\mathrm{cmc}$ of the individual components reflecting their relative tendency toward micellization in the mixed state. The theory is, therefore, an oversimplification and can be applied only in fairly dilute solutions (systems with very low $\mathrm{cmc}$ ). The theory is, however, a tool, divergence from which in the positive and negative side signifies antagonistic and synergistic behavior, respectively. It is clear from the data (Tables 1-3) that the cmc values of the mixture of gemini+conventional surfactants are intermediate to the component $\mathrm{cmc}$ at all mole fractions. This shows that the gemini surfactants can partition easily into the micelles of monomeric surfactants and strengthen the hydrophobic environment in the mixed state in comparison to pure state. Therefore, micellization takes place at lower concentrations as compared to the ideal state. One can see (Figs.3 and 4) that the cmc values are always smaller than the $\mathrm{cmc}^{*}$ values and the difference between $\mathrm{cmc}$ and cmc* increases with the increase in mole fraction of the conventional surfactant. A lower $\mathrm{cmc}$ value than the corresponding cmc $^{*}$ indicates that mixed micellization is due to some sort of attractive interactions synergism operating between the two components of the mixtures.

The fraction of counterions associated with the Stern part of the double layer is termed as counterion binding. Ionic micelles bind a considerable amount of counterions, which can be estimated by electrochemical measurements. All aggregation processes in aqueous solutions can be understood by the counterion binding. The degree of counterion binding depends on surface

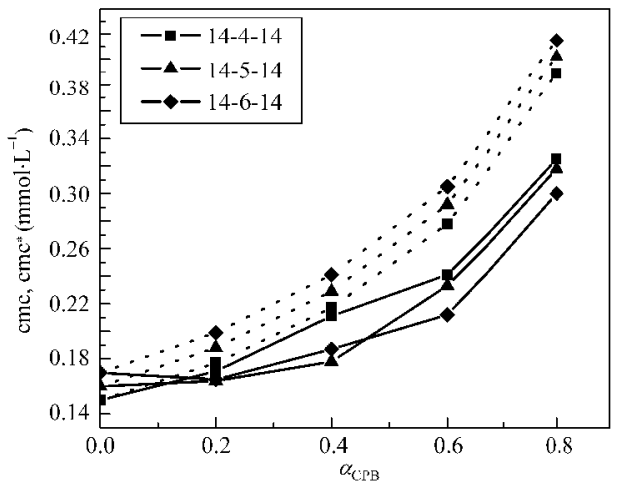

Fig.3 Critical micelle concentrations for binary mixtures of 14-s-14+CPB

Solid lines represent experimental data $(\mathrm{cmc})$ and dashed lines were calculated from Clint's model $\left(\mathrm{cmc}^{*}\right)$.

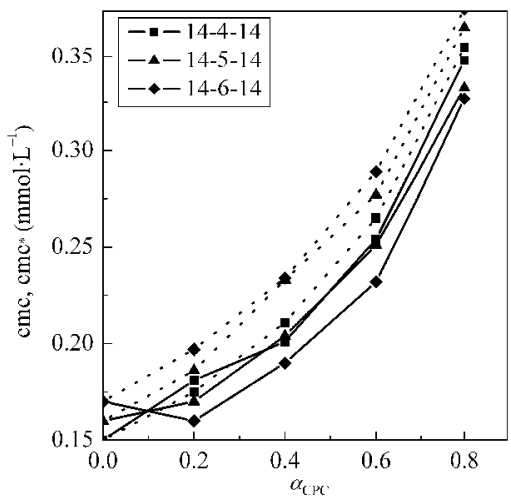

Fig.4 Critical micelle concentrations for binary mixtures of 14-s-14+CPC

Solid lines represent experimental data $(\mathrm{cmc})$ and dashed lines were calculated from Clint's model $\left(\mathrm{cmc}^{*}\right)$.

charge density and polarizibility. The counterion binding in creases with increase in the polarizibility and charge density. The cmc of ionic surfactants is also affected by counterion binding, an increase in the cmc decreases the counterion binding. The counterion binding of the mixed systems decreases in all the cases with increasing the mole fraction of component $1\left(\alpha_{1}\right.$, Tables 1-3), which suggests loose micellar aggregates.

In order to investigate the nature of interactions among the components, we calculated various other parameters, using Rubingh's model ${ }^{[13]}$.

The Rubingh model is the first model developed for nonideal mixed systems. It is based on regular solution approach for the treatment of a nonideal mixing, and due to its simplicity, it has been mostly used, even after development of more complex models. The nonideality is introduced with the inclusion of the activity coefficients $\left(f_{i}\right)$ in equation (2), i.e.,

$$
\frac{1}{\mathrm{cmc}^{*}}=\sum_{i=1}^{n} \frac{\alpha_{i}}{f_{i} \mathrm{cmc}_{i}}
$$

where, in the case of a binary solution,

$$
f_{1}=\exp \left[\beta\left(1-X_{1}\right)^{2}\right]
$$

and

$$
f_{2}=\exp \left(\beta X_{1}^{2}\right)
$$

The $\beta$ parameter can be interpreted in terms of an energetic parameter that represents the Gibbs excess free energy of mixing, which, according to the regular solution approximation, is true only in the case when the excess entropy of mixing is zero. The $\beta$ parameter can be determined from experimental values of $\mathrm{cmc}$ using the following equations

$$
\begin{aligned}
& \beta=\left[\ln \left(\alpha_{1} \mathrm{cmc} / X_{1} \mathrm{cmc}_{1}\right)\right] /\left(1-X_{1}\right)^{2} \\
& \frac{\left[X_{1}^{2} \ln \left(\alpha_{1} \mathrm{cmc}_{X_{1}} \mathrm{cmc}_{1}\right)\right]}{\left(1-X_{1}\right)^{2} \ln \left[\mathrm{cmc}\left(1-\alpha_{1}\right) / \mathrm{cmc}_{2}\left(1-X_{1}\right)\right]}=1
\end{aligned}
$$

where $X_{1}$ is the micelle mole fraction of surfactant 1 (i.e., conventional) in the mixed micelle. Equation (8) was solved iteratively to obtain the values of $X_{1}$. The mole fraction of a particular component in the micelle is lower than that of stoichiometric mole fraction of that component (Tables 1-3) indicating the low extent of transfer of that component from solution to micelle. The micelle mole fraction in the ideal state $\left(X_{1}^{\text {ideal }}\right)$ was also evaluated by 
applying Motomura's theory ${ }^{[15]}$, which is based upon excess thermodynamic quantities, as

$X_{1}^{\text {ideal }}=\left[\left(\alpha_{1} \mathrm{cmc}_{2}\right) /\left(\alpha_{1} \mathrm{cmc}_{2}+\left(1-\alpha_{1}\right) \mathrm{cmc}_{1}\right)\right]$

It is clear from the data (Tables $1-3$ ) that $X_{1}$ values are always more than $X_{1}^{\text {ideal }}$, which means that, even in a low conventional surfactant region (i.e., with low $\alpha_{1}$ values), the mixed micelles are rich in conventional surfactants in comparison to that in ideal mixing state (i.e., $X_{1}^{\text {ideal }}$ ). Further, as the length of spacer $(s)$ increases from 4 to 6 , the value of $X_{1}$ becomes larger and the difference between $X_{1}$ and $X_{1}^{\text {ideal }}$ values increases. This means that at the same mixture composition, as the spacer chain length changes from 4 to 6 , the contribution of gemini surfactant decreases and gemini molecules contribute less as compared to their ideal state.

The mixing of hydrophobic core to hydrophobic chain of a binary mixture leads to ideal process while electrostatic interaction between head groups leads to nonideal process. Therefore, even the monomer phase in micelle solution is considered to be ideal, the micellar phase may be nonideal. The nonideality can be expressed in terms of an interaction parameter $\beta$, which corepresents the free energy of mixing. For most of the systems where hydrocarbon chains mix inside the hydrophobic micelle core, $\beta$ is negative. This means that the free energy is lowered when a hydrocarbon chain moves from the monomer phase to the micelle phase. A zero value indicates ideal mixing while positive values show less attraction after mixing than before mixing (antagonism)

In our case, as can be seen from the data, the average values of interaction parameter, $\beta_{\mathrm{av}}$, come out to be $-0.532 /-1.280$ / -1.600 and $-0.264 /-0.607 /-1.149$ for $14-4-14 / 14-5-14 / 14-6-14$ geminis with $\mathrm{CPB}$ and $\mathrm{CPC}$, respectively. All the values being negative indicate attractive interaction among the gemini and conventional surfactants in mixed micelles. Increase in spacer length also affects synergistic interactions, most likely due to the increase in hydrophobocity at the level of head group and the modification in the distance between head groups of amphiphiles in the micellar phase. Since there are two long hydrophobic chains in the geminis, too small a linkage (less hydrophobic) between the two head groups is not suitable for their packing and inhibits their interaction with other surfactants at the surface of the convex micelle and results in a low average value of $\beta$.

The values of activity coefficients, $f_{1}$ and $f_{2}$, calculated from equations (5) and (6), are found to be less than unity showing nonideal behavior of the mixed systems. The activity coefficients were used to calculate excess free energy of mixing by the relation

$$
\Delta G_{\mathrm{ex}}=R T\left[X_{1} \ln f_{1}+\left(1-X_{1}\right) \ln f_{2}\right]
$$

The negative $\Delta G_{\text {ex }}$ values thus obtained (Tables 1-3) suggest that the mixed micelles are more stable than the micelles of indi- vidual components.

\section{Conclusions}

Interaction between cationic gemini and cationic conventional surfactants was investigated at $303 \mathrm{~K}$. The following conclusions were obtained:

(1) The experimental cmc values of mixtures are lower than those predicted from Clint's equation, indicating the presence of nonideality in the systems.

(2) In this study, the mixed micelle is also interpreted theoretically. The mole fraction of a particular component in the micelle is lower than that of stoichiometric mole fraction of that component indicating the low extent of transfer of that component from solution to micelle.

(3) The binary mixtures show synergism (negative $\beta$ values). On increasing the hydrophobicity of the spacer the sysnergistic interaction also increases, it is also confirmed by the average excess free energy.

\section{References}

1 Menger, F. M.; Littau, C. A. J. Am. Chem. Soc., 1991, 113: 1451

2 Menger, F. M.; Littau, C. A. J. Am. Chem. Soc., 1993, 115: 10083

3 Rosen, M. J. Chemtech, 1993, 23: 30

4 Junger, E. Cationic surfactants. New York: Marcel Dekker, 1970

5 Aungst, B. J.; Phang, S. Int. J. Pharm., 1995, 117: 95

6 Abe, M.; Ogino, K. Mixed surfactant systems. New York: Marcel Dekker, 1993

7 Gracia, M. T.; Ribosa, I.; Leal, J. S.; Comelles, F. J. Am. Oil Chem. Soc., 1992, 69: 25

8 Kibbey, T. C. G.; Hayes, K. F. Environ. Sci. Tech., 1997, 3: 1171

9 Junquera, E.; Pea, L.; Aicart, E. Langmuir, 1997, 13: 219

10 Zhou, Q.; Rosen, M. J. Langmuir, 2003, 19: 4555

11 Clint, J. H. J. Chem. Soc. Faraday Trans. 1, 1975, 71: 1327

12 Clint, J. H. Surfactant aggregation. New York: Chapman and Hall, 1992

13 Holland, P. M.; Rubingh, D. N. J. Phys. Chem., 1983, 87: 1984

14 Gu, B.; Rosen, M. J. J. Colloid Interface Sci., 1989, 129: 537

15 Motomura, K.; Yamanaka, M.; Aratono, M. Colloid Polym. Sci., 1984, 262: 948

16 Sarmoria, C.; Puvvada, S.; Blankschtein, D. Langmuir, 1992, 8: 2690

17 Puvvada, S.; Blankschtein, D. J. Phys. Chem., 1992, 96: 5567

18 Puvvada, S.; Blankschtein, D. J. Phys. Chem., 1992, 96: 5579

19 Maeda, H. J. Colloid Interface Sci., 1995, 98 : 172

20 De, S.; Aswal, V. K.; Goyal, P. S.; Bhattacharya, S. J. Phys. Chem., 1996, 100: 11664

21 Tanford, C. The hydrophobic effect: formation of micelles and biological membranes. New York: Wiley, 1980 